\title{
Heat Kernel Regularization of Quantum Fields *
}

Arthur Jaffe, Andrzej Lesniewski, and Christian Wieczerkowski ${ }^{\star \star}$

Lyman Laboratory of Physics, Harvard University, Cambridge, MA 02138, USA

\begin{abstract}
We discuss some consequences of the existence of a heat kernel regularization (HKR) for quantum fields. We demonstrate that HKR applies in certain examples, using methods which should be useful more generally.
\end{abstract}

\section{The Heat Kernel Regularization}

Let $\mathscr{H}$ be a Hilbert space and let $H \geqq 0$ be a self-adjoint operator. We consider the self-adjoint contraction semigroup $T(t)=\exp (-t H), t \geqq 0$, which is generated by $H$. We introduce a scale of Hilbert spaces

$$
\mathscr{H}_{\varepsilon} \rightarrow \mathscr{H}^{\rightarrow} \mathscr{H}_{-\varepsilon}, \quad \varepsilon>0,
$$

where $\mathscr{H}_{\varepsilon}$ is the completion of the domain of $\exp (\varepsilon H)$ with respect to the inner product

$$
\left\langle\chi_{1}, \chi_{2}\right\rangle_{\mathscr{H}_{\varepsilon}}=\left\langle e^{\varepsilon H} \chi_{1}, e^{\varepsilon H} \chi_{2}\right\rangle_{\mathscr{H}} .
$$

Also, $\mathscr{H}_{-\varepsilon}$ is the dual space to $\mathscr{H}_{\varepsilon}$ with respect to $\langle\cdot, \cdot\rangle_{\mathscr{H}}$. By $\mathscr{L}\left(\mathscr{H}_{\varepsilon}, \mathscr{H}_{-\varepsilon}\right)$ we denote the space of bounded linear operators mapping $\mathscr{H}_{\varepsilon}$ into $\mathscr{H}_{-\varepsilon}$. Let $W$ be a bilinear form on $\mathscr{H}$ with domain $\mathscr{H}_{\varepsilon} \times \mathscr{H}_{\varepsilon}$ for some $\varepsilon>0$.

Definition I.1. We say that $W$ has a heat kernel regularization (HKR), if

$$
W \in \mathscr{L}\left(\mathscr{H}_{\varepsilon}, \mathscr{H}_{-\varepsilon}\right) \text {. }
$$

Furthermore, if (I.3) holds for every $\varepsilon>0$, then we say that $W$ has a strong HKR.

Let

$$
W_{\varepsilon}=e^{-\varepsilon H} W e^{-\varepsilon H}
$$

denote the operator on $\mathscr{H}$ uniquely determined by (I.3). We call $W_{\varepsilon}$ the HKR of $W$. The condition (I.3) is equivalent to the boundedness of $W_{\varepsilon}$ on $\mathscr{H}$,

$$
W_{\varepsilon} \in \mathscr{L}(\mathscr{H}, \mathscr{H}) \Leftrightarrow W \in \mathscr{L}\left(\mathscr{H}_{\varepsilon}, \mathscr{H}_{-\varepsilon}\right) .
$$

* Supported in part by the National Science Foundation under Grant PHY/DMS 86-45122

** Supported in part by a German National Scholarship Foundation fellowship 
The notion of heat kernel regularization is particularly natural in analysis on infinite dimensional manifolds, e.g., in the context of quantum field theory [1]. The existence of a HKR in quantum field theory is a stronger property then the domain assumptions in the standard Wightman axioms, see e.g. [2]. Its advantage is that it provides a clean treatment of the operator theory for local quantum fields. Since the use of the heat kernel has become a standard way to investigate quantum fields, and since it is often combined with a path integral representation to establish estimates, the study of HKR is quite natural.

In this paper we discuss the HKR property in the context of the $N=1$ and $N=2$ two-dimensional Wess-Zumino models (see [3] for a review). We prove that Wick polynomials in the local field operators have a strong HKR. Also, we show that this fact and the spectral condition lead to certain analyticity properties of the heat kernel regularized field operators.

\section{Regularity of Wick Polynomials}

In this section we establish boundedness of heat kernel regularized Wick polynomials in the time-zero field operators. Such Wick polynomials are densely defined bilinear forms on the Fock space $\mathscr{H}$. Our results show that they define bounded operators from $\mathscr{H}_{\varepsilon}$ to $\mathscr{H}_{-\varepsilon}$ for $\varepsilon>0$.

We prove the estimates for the special class of models studied in [4-6]. The same methods apply to the models presented in [2], and in principle to all other models studied in constructive field theory. Without loss of generality we can restrict attention to Wick monomials; polynomials are defined by linearity. Let $\varphi^{\#}$ denote $\varphi$ or $\varphi^{*}$; in the $N=1$ case $\varphi^{\#}=\varphi$ as the boson is self-adjoint. Likewise, let $\psi^{\prime \#}$ denote $\psi$ or $\bar{\psi}=\psi^{*} \gamma_{0}$, where $\gamma_{0}=\left(\begin{array}{ll}0 & 1 \\ 1 & 0\end{array}\right)$. Consider the circle $T^{1}=S^{1}=\mathbb{R} / l \mathbb{Z}$ and let $\underline{x}=\left(x_{1}, \ldots, x_{b}\right), \underline{y}=\left(y_{1}, \ldots, y_{f}\right)$ be points in $\left(T^{1}\right)^{b}$ or $\left(T^{1}\right)^{f}$. The Wick monomials

$$
W(\underline{x}, \underline{y})=: \prod_{j=1}^{b} \varphi^{\#}\left(x_{j}\right):: \prod_{j=1}^{f} \psi_{\alpha_{j}}^{\#}\left(y_{j}\right):
$$

are defined in [7] as densely defined bilinear forms on Fock space. Here the product over fermions is taken, by convention, from left to right in order of increasing $j$. Also let

$$
W_{\varepsilon}(\underline{x}, \underline{y})=e^{-\varepsilon H} W(\underline{x}, \underline{y}) e^{-\varepsilon H},
$$

where $H$ is the Hamiltonian of the $N=1$ or $N=2$ models studied in [4-6]. Let $\|\cdot\|_{p}$ denote the $p^{\text {th }}$ Schatten norm. Our main result is

Theorem II.1. For $\varepsilon>0$, the bilinear form $W_{\varepsilon}(\underline{x}, \underline{y})$ defines a Hilbert-Schmidt operator on $\mathscr{H}$, and

$$
\left\|W_{\varepsilon}(\underline{x}, \underline{y})\right\|_{2} \leqq C<\infty
$$

with $C$ independent of $(\underline{x}, \underline{y})$.

Remark. The proof we give can easily be modified to prove the same result in all previously constructed, two-dimensional models.

The remainder of this section is devoted to the proof of (II.3). We use a path space (Feynman-Kac) representation of $\left\|W_{\varepsilon}(\underline{x}, \underline{y})\right\|_{2}^{2}$. Such representations are 
standard in constructive field theory and were used in $[5,6]$ to establish the a priori estimates leading to the existence of a theory. Our notation follows [3-6]. We give a detailed proof of Theorem II. 1 for the $N=2$ models. The case of the $N=1$ models is treated similarly. The only difference is that our estimates on determinants have to be replaced by estimates on Pfaffians along the lines of $[6,8]$.

Let us introduce a regularized approximation to $W_{\varepsilon}$, namely

$$
W_{\varepsilon}^{(\kappa)}(\underline{x}, \underline{y})=e^{-\varepsilon H(\kappa)} W(\underline{x}, \underline{y}) e^{-\varepsilon H(\kappa)},
$$

with $H(\kappa)$ defined in [4-6]. For $\kappa<\infty$, the Hamiltonian $H(\kappa)$ involves finitely many interacting modes, so it is easy to see that $W_{\varepsilon}^{(\kappa)}(\underline{x}, \underline{y})$ is Hilbert-Schmidt. Using standard methods we obtain the Feynman-Kac representation of

$$
\left\|W_{\varepsilon / 2}^{(\kappa)}(\underline{x}, \underline{y})-W_{\varepsilon / 2}^{\left(\kappa^{\prime}\right)}(\underline{x}, \underline{y})\right\|_{2}^{2} .
$$

This representation is a sum of terms of the following form. The first term is

where

$$
\begin{aligned}
& \Xi_{l, 2 \varepsilon} \int^{3}: \prod_{j=1}^{b} \phi^{\#}\left(x_{j}, 0\right)^{*} \prod_{j=1}^{b} \phi^{\#}\left(x_{j}, \varepsilon\right): \\
& \quad \times\left[F_{l, 2 \varepsilon}^{(\kappa)}\left(\underline{y} ; \phi, \phi^{*}\right)+F_{l, 2 \varepsilon}^{\left(\kappa^{\prime}\right)}\left(\underline{y} ; \phi, \phi^{*}\right)-2 F_{l, 2 \varepsilon}^{\left(\kappa, \kappa^{\prime}\right)}\left(\underline{y} ; \phi, \phi^{*}\right)\right] d \mu_{c}\left(\phi, \phi^{*}\right),
\end{aligned}
$$

$$
\begin{aligned}
F_{l, 2 \varepsilon}^{(\kappa)}\left(y ; \phi, \phi^{*}\right)= & (-1)^{\#} \operatorname{det}\left\{\left(I-\widetilde{K}_{l, 2 \varepsilon}^{(\kappa)}\left(\phi, \phi^{*}\right)\right)^{-1} \widetilde{K}_{l .2 \varepsilon}^{(\kappa)}\left(\phi, \phi^{*}\right) \widetilde{S}_{\sigma_{J} \tau_{k}}\left(w_{j}, z_{k}\right)\right\} \\
& \times \operatorname{det}_{3}\left(I-\widetilde{K}_{l, 2 \varepsilon}^{(\kappa)}\left(\phi, \phi^{*}\right)\right) \exp \left\{-\tilde{\mathscr{A}}_{l .2 \varepsilon}^{(\kappa)}\left(\phi, \phi^{*}\right)\right\},
\end{aligned}
$$

with the sign $(-1)^{\#}$ depending on the choice of \#, and where $F_{l, 2 \varepsilon}^{\left(\kappa, \kappa^{\prime}\right)}$ is defined by a similar expression, but with $\widetilde{K}_{l, 2 \varepsilon}^{(\kappa)}$ and $\tilde{\mathscr{A}}_{l, 2 \varepsilon}^{(\kappa)}$ replaced by $\tilde{K}_{l, 2 \varepsilon}^{\left(\kappa, \kappa^{\prime}\right)}$ and $\tilde{\mathscr{A}}_{l, 2 \varepsilon}^{\left(\kappa, \kappa^{\prime}\right)}$, respectively. (See [5], Eqs. (41-2) for the definitions.) The first determinant in (II.7) is the determinant of an $f \times f$ matrix, while $\operatorname{det}_{3}$ is the regularized Fredholm determinant. The fact that the entry of the finite dimensional determinant in (II.7) has a factor $\widetilde{K}_{l, 2 \varepsilon}^{(\kappa)}\left(\phi, \phi^{*}\right)$ is the result of the Wick ordering of the fermions. Also, $w_{1}, \ldots, w_{f}, z_{1}, \ldots, z_{f}$ is a permutation of $\left(y_{1}, 0\right), \ldots,\left(y_{f}, 0\right),\left(y_{1}, \varepsilon\right), \ldots,\left(y_{f}, \varepsilon\right)$, whose only property relevant for the arguments to follow is that $\left|\left(w_{j}\right)_{2}-\left(z_{k}\right)_{2}\right|=\varepsilon$, where $\left(w_{j}\right)_{2}$ is the second coordinate of $w_{j}$. The sign $(-1)^{\#}$ plays no role in our proof. The other terms in the Feynman-Kac representation of (II.5) have the form of a product of a smooth function in a subset of variables $\underline{x}, \underline{y}$ and an expression of the form (II.6) (with some other $b$ and $f$ ). It is thus sufficient to study (II.6).

We showed in [5] that

$$
F_{l, \beta}\left(\phi, \phi^{*}\right)=\operatorname{det}_{3}\left(I-\tilde{K}_{l, \beta}\left(\phi, \phi^{*}\right)\right) \exp \left\{-\tilde{\mathscr{A}}_{l, \beta}\left(\phi, \phi^{*}\right)\right\},
$$

(i.e., $\kappa=\infty$ ) is $L_{p}$, for each $1 \leqq p<\infty$. The only new difficulties arise from the factors $(I-K)^{-1} K S$. We consider

$$
\begin{aligned}
F_{l, \beta}\left(\underline{y} ; \phi, \phi^{*}\right)= & (-1)^{\#} \operatorname{det}\left\{\left(I-\tilde{K}_{l, \beta}\left(\phi, \phi^{*}\right)\right)^{-1} \tilde{K}_{l, \beta}\left(\phi, \phi^{*}\right)\right. \\
& \left.\times \tilde{S}_{\sigma_{J} \tau_{k}}\left(w_{j}, z_{k}\right)\right\} F_{l, \beta}\left(\phi, \phi^{*}\right) .
\end{aligned}
$$

Proposition II.2. For $\varepsilon>0$,

$$
\left|\int: \prod_{j=1}^{b} \phi^{\#}\left(x_{j}, 0\right)^{*} \prod_{j=1}^{b} \phi\left(x_{j}, \varepsilon\right): F_{l, \beta}\left(\underline{y} ; \phi, \phi^{*}\right) d \mu_{C}\left(\phi, \phi^{*}\right)\right| \leqq C<\infty,
$$

uniformly in $(\underline{x}, \underline{y})$. 
Proof. Using Gaussian integration by parts, we see that the integral in (II.10) equals

$$
\begin{aligned}
& \int\left\{\prod_{j} \frac{\delta}{\delta \phi^{\#}\left(u_{j}\right)^{*}} \prod_{j} \frac{\delta}{\delta \phi^{\#}\left(v_{j}\right)} F_{l, \beta}\left(y ; \phi, \phi^{*}\right)\right. \\
& \left.\quad \times \prod_{j} C\left(\left(x_{j}, \varepsilon\right)-u_{j}\right) \prod_{j} C\left(\left(x_{j}, 0\right)-v_{j}\right) d \underline{u} d \underline{v}\right\} d \mu_{C}\left(\phi, \phi^{*}\right) .
\end{aligned}
$$

To estimate (II.11) we apply Cauchy's bound. Let $f, g \in L_{p}\left(T^{2}\right)$ for all $1 \leqq p<\infty$. For $\varrho \in L_{\infty}\left(d \mu_{C}\right)$ we define

$$
\mathscr{F}(\zeta, \eta ; \varrho)=\int \varrho\left(\phi, \phi^{*}\right) F_{l, \beta}\left(\underline{y} ; \phi+\zeta f, \phi^{*}+\eta g\right) d \mu_{C}\left(\phi, \phi^{*}\right),
$$

where $(\zeta, \eta) \in \mathbb{C}^{2}$. Let $D_{r} \subset \mathbb{C}^{2}$ denote a closed polydisc of radius $r$ around the origin.

Lemma II.3. For $\varrho \in L_{\infty}$, the $\operatorname{map}(\zeta, \eta) \rightarrow \mathscr{F}(\zeta, \eta ; \varrho)$ is entire. Furthermore,

$$
\sup _{(\zeta, \eta) \in D_{r}}\|\mathscr{F}(\zeta, \eta ; \varrho)\| \leqq C_{r}\|\varrho\|_{L_{\infty}}
$$

uniformly in $(\underline{x}, \underline{y})$.

Assuming the lemma, it follows by Cauchy's bound that for $(\zeta, \eta) \in D_{r}$,

$$
\left|\frac{\partial}{\partial} \tilde{\zeta} \mathscr{F}(\zeta, \eta ; \varrho)\right| \leqq C_{r}\|\varrho\|_{L_{\infty}} .
$$

We infer that the $L_{1}$-derivative

$$
\frac{\partial}{\partial \zeta} F_{l, \beta}\left(\underline{y} ; \phi+\zeta f, \phi^{*}+\eta g\right)
$$

exists, and that

$$
\frac{\partial}{\partial \zeta} \mathscr{F}(\zeta, \eta ; \varrho)=\int \varrho\left(\phi, \phi^{*}\right) \frac{\partial}{\partial \zeta} F_{l, \beta}\left(\underline{y} ; \phi+\zeta f, \phi^{*}+\eta g\right) d \mu_{C}\left(\phi, \phi^{*}\right) .
$$

Analogous statements hold for $\partial / \partial \eta$. As a consequence, (II.11) can be written in the form

$$
\left.\left(\prod_{j \in S_{1} \cup S_{2}} \frac{\partial}{\partial \zeta_{j}}\right) \int F_{l, \beta}\left(\underline{y} ; \phi+\sum_{j \in S_{1}} \zeta_{j} f_{j}, \phi^{*}+\sum_{j \in S_{2}} \zeta_{j} f_{j}\right) d \mu_{C}\right|_{\xi=\overline{0}},
$$

where $f_{j}(z)=C\left(v_{j}-z\right) \in L_{p}\left(T^{2}\right), 1 \leqq p<\infty$. Using Lemma II.3 and Cauchy's bound we obtain (II.10), choosing $\varrho$ constant.

Proof of Lemma II.3. Let $u(\zeta, \bar{\zeta}, \eta, \bar{\eta})$ be a smooth function supported in $D_{r}$. We show that

$$
\left\|F_{l, \beta}\left(\underline{y} ; \phi+\zeta f, \phi^{*}+\eta g\right)\right\|_{L_{2}\left(d \mu_{C}\right)} \leqq C<\infty,
$$

uniformly in $y$ and $(\zeta, \eta) \in D_{r}$. Note that $F_{l, \beta}$ depends implicitly also on $\underline{x}$ through our choice of $f_{j}$. It is clear that the estimate (II.15) holds uniformly in $\underline{x}$. This estimate implies that the function

$$
u(\zeta, \bar{\zeta}, \eta, \bar{\eta}) \varrho\left(\phi, \phi^{*}\right) F_{l, \beta}\left(\underline{y} ; \phi+\zeta f, \phi^{*}+\eta g\right)
$$

defined on $\mathbb{C}^{2} \times \mathscr{S}^{\prime}\left(T^{2}\right)$ is $L_{1}\left(\mathbb{C}^{2} \times \mathscr{S}^{\prime}\left(T^{2}\right),|d \zeta d \bar{\zeta} d \eta d \bar{\eta}| \otimes d \mu_{C}\right)$. Therefore, the weak derivatives $\partial / \partial \bar{\zeta}$ and $\partial / \partial \bar{\eta}$ of $\mathscr{F}(\zeta, \eta ; \varrho)$ are zero. By the elliptic regularity theorem, $\mathscr{F}(\zeta, \eta ; \varrho)$ is holomorphic. Estimate (II.13) is a consequence of (II.15). 
To prove (II.15) it is instructive to consider first the case of $F_{l, \beta}\left(\phi, \phi^{*}\right)$ (no fermion operators). As shown in [5],

$$
\left|F_{l, \beta}\left(\phi, \phi^{*}\right)\right| \leqq R\left(\phi, \phi^{*}\right),
$$

where

$$
R=\left\{\operatorname{det}_{4}\left(I+L_{+}\right)\right\}^{1 / 2} \exp (-\mathscr{W}),
$$

where $L_{+}$is the positive part of the operator $L=-K-K^{*}+K^{*} K$, and where

$$
\mathscr{W}=\mathscr{A}+\frac{1}{4}\|K\|_{4}^{4}-\operatorname{Re} \operatorname{Tr}\left(K^{2} K^{*}\right)-\frac{1}{6} \operatorname{Tr} L^{3} .
$$

We show that for $1 \leqq p<\infty$,

$$
\sup _{(\zeta, \eta) \in D_{r}}\left\|R\left(\phi+\zeta f, \phi^{*}+\eta g\right)\right\|_{L_{p}} \leqq C<\infty .
$$

To prove (II.20) we proceed as in $[9,5]$. We introduce an auxiliary regularization $\lambda>0$. Let $\phi_{\lambda}=\chi_{2} * \phi$, where $\chi_{\lambda}$ is a cutoff function. Denoting

$$
v\left(\phi, \phi^{*}\right)=\log R\left(\phi, \phi^{*}\right),
$$

and using $f, g \in L_{p}\left(T^{2}\right), 1 \leqq p<\infty$, we establish (as in [5]) the following bounds:

$$
v\left(\phi_{\lambda}+\zeta f, \phi_{\lambda}^{*}+\eta g\right) \geqq C \int\left|\phi_{\lambda}(x)\right|^{2(n-1)} d x-O\left((\log \lambda)^{n-1}\right),
$$

and

$$
\left\|v\left(\phi+\zeta f, \phi^{*}+\eta g\right)-v\left(\phi_{\lambda}+\zeta f, \phi_{\lambda}^{*}+\eta g\right)\right\|_{L_{2}} \leqq O\left(\lambda^{-\delta}\right),
$$

for some $\delta>0$, uniformly in $(\zeta, \eta) \in D_{r}$. These two bounds yield (II.20).

We return now to the general case. Write

$$
(I-K)^{-1} K=K+K(I-K)^{-1} K,
$$

in (II.9). Then the finite dimensional determinant is a sum of terms of the following form:

$$
\begin{aligned}
& \pm \operatorname{det} \mid \begin{array}{cc}
K S\left(w_{1}-z_{1}\right) \ldots K S\left(w_{1}, z_{p}\right) \\
\vdots & \vdots \\
K S\left(w_{m}-z_{1}\right) \ldots K S\left(w_{m, z_{p}}\right)
\end{array} \\
& \left.\begin{array}{cc}
K(1-K)^{-1} K S\left(w_{1}, z_{p+1}\right) \ldots K(I-K)^{-1} K S\left(w_{1}, z_{m}\right) \\
\vdots & \vdots \\
K(I-K)^{-1} K S\left(w_{m}, z_{p+1}\right) \ldots K(I-K)^{-1} K S\left(w_{m}, z_{m}\right)
\end{array}\right) .
\end{aligned}
$$

Here the first $p$ columns have the kernels $K S(w, z)$ as the entries, and the last $m-p$ columns consist of the kernels $K(I-K)^{-1} K S(w, z)$. Expanding (II.25) with respect to the first $p$ columns, we obtain a sum of terms of the form

$$
\pm\left(\prod K S\left(w_{a}, z_{b}\right)\right) \operatorname{det}\left\{K(I-K)^{-1} K S\left(w_{j}, z_{k}\right)\right\} .
$$

We use Hölder's inequality to obtain

$$
\begin{aligned}
& \int\left|\left(\prod K S\left(w_{a}, z_{b}\right)\right) \operatorname{det}\left\{K(I-K)^{-1} K S\left(w_{i}, z_{k}\right)\right\} \operatorname{det}_{3}(I-K) \exp (-\mathscr{A})\right|^{2} d \mu_{C} \\
& \quad \leqq \prod\left\|K S\left(w_{a}, z_{j}\right)\right\|_{L_{4}}^{2}\left\|\operatorname{det}\left\{K(I-K)^{-1} K S\left(w_{j}, z_{k}\right)\right\} \operatorname{det}_{3}(I-K) \exp (-\mathscr{A})\right\|_{L_{4}}^{2} .
\end{aligned}
$$


By hypercontractivity, this is bounded if the following two estimates hold:

$$
\|K S(w, z)\|_{L_{2}} \leqq C,
$$

and

$$
\left\|\operatorname{det}\left\{K(I-K)^{-1} K S\left(w_{j}, z_{k}\right)\right\} \operatorname{det}_{3}(I-K) \exp (-\mathscr{A})\right\|_{L^{2}} \leqq C,
$$

uniformly in $(\zeta, \eta) \in D_{r}$. To prove these two estimates we need the following.

Lemma II.4. (i) Let $h \in L_{2}\left(T^{2}\right)$ and let $\phi^{j}(h)=\int \phi(z)^{j} h(z) d z$. Then

$$
\left\|\phi^{j}(h)\right\|_{L_{2}} \leqq C\|h\|_{L_{2}\left(T^{2}\right)} .
$$

(ii) Let $U_{j}(w, z)$ and $V_{j}(w, z)$ satisfy $\left\|U_{j}\right\|_{*}<\infty,\left\|V_{j}\right\|_{*}<\infty$, where $\left\|_{*}\right\|_{*}$ denotes the negative Sobolev space norm

$$
\|U\|_{*}^{2}=\int\left|\left(-\Delta_{w}+m^{2}\right)^{-1 / 4} U(w, z)\right|^{2} d w d z .
$$

Set

$$
\begin{aligned}
& \alpha_{j}(w)=\int U_{j}(w, z): \phi^{\#}(z)^{k_{j}}: d z, \\
& \beta_{j}(w)=\int V_{j}(w, z): \phi^{\#}(z)^{l_{j}}: d z .
\end{aligned}
$$

Then

$$
\begin{aligned}
& \|\left(\alpha_{1} \wedge \ldots \wedge \alpha_{n}, \wedge^{n}\left(I-\tilde{K}_{l, \beta}\left(\phi+\zeta f, \phi^{*}+\eta g\right)\right)^{-1} \widetilde{S}_{l, \beta} \beta_{1} \wedge \ldots \wedge \beta_{n}\right) \\
& \quad \times F_{l, \beta}\left(\phi+\zeta f, \phi^{*}+\eta g\right)\left\|_{L_{2}} \leqq C\right\| R\left(\phi+\zeta f, \phi^{*}+\eta g\right)\left\|_{L_{2}} \prod_{j=1}^{n}\right\| U_{j}\left\|_{*}\right\| V_{j} \|_{*},
\end{aligned}
$$

for $(\zeta, \eta) \in D_{r}$.

Proof. (i) This is a standard estimate which uses the fact that $C_{l, \beta}(w-z) \sim \log |w-z|$, as $|w-z| \rightarrow 0$. (ii) Using the bound (see e.g. [5], Sect. IV)

$$
\begin{aligned}
& \left|\left(\alpha_{1} \wedge \ldots \wedge \alpha_{n}, \wedge^{n}(I-K)^{-1} S \beta_{1} \wedge \ldots \wedge \beta_{n}\right) \operatorname{det}_{3}(I-K) \exp (-\mathscr{A})\right| \\
& \quad \leqq C R \prod_{j=1}^{n}\left\|\alpha_{j}\right\|_{\mathscr{H}-1 / 2}\left\|\beta_{j}\right\|_{\mathscr{H}-1 / 2},
\end{aligned}
$$

and Hölder's inequality, we bound the left-hand side of (II.31) by

$$
\begin{aligned}
& C \prod_{j=1}^{n}\left\|\int\left|\int\left(-\Delta_{w}+m^{2}\right)^{1 / 4} U_{j}(w, z): \phi^{\#}(z)^{k_{j}}: d z\right|^{2} d w\right\|_{L_{4 n}} \\
& \quad \times \prod_{j=1}^{n}\left\|\int\left|\int\left(-\Delta_{w}+m^{2}\right)^{1 / 4} V_{j}(w, z): \phi^{\#}(z)^{l_{j}}: d z\right|^{2} d w\right\|_{L_{4 n}} \\
& \quad \times\left\|R\left(\phi+\zeta f, \phi^{*}+\eta g\right)\right\|_{L_{4}} .
\end{aligned}
$$

Using hypercontractivity we replace the $L_{p}$-norms by the $L_{2}$-norm in the above expression. Since

$$
\left\|\int\left|\int k(w, z) \phi^{\#}(z)^{j} d z\right|^{2} d w\right\|_{L_{2}} \leqq C\|k\|_{L_{2}\left(T^{2} \times T^{2}\right)}^{2},
$$

inequality (II.31) follows. 
To prove (II.26) we apply (II.28) with

$$
h(x)=\zeta^{j} f(x)^{j} S(w-x) S(x-z)
$$

or

$$
h(x)=\eta^{j} g(x)^{j} S(w-x) S(x-z) .
$$

To prove (II.27) we apply (II.31) with $U$ and $V$ of the form $\zeta^{j} S(w-z) f(z)^{j}$ or $\eta^{j} S(w-z) g(z)^{j}$. The proof of Proposition II.2 is complete.

Proposition II.5. For fixed $\varepsilon>0$,

(i)

$$
\int: \prod_{j=1}^{b} \phi^{\#}\left(x_{j}, 0\right)^{*} \prod_{j=1}^{b} \phi^{\#}\left(x_{j}, \varepsilon\right):\left[F_{l, 2 \varepsilon}^{(\kappa)}\left(\underline{y} ; \phi, \phi^{*}\right)-F_{l, 2 \varepsilon}\left(\underline{y} ; \phi, \phi^{*}\right)\right] d \mu_{\mathrm{C}}\left(\phi, \phi^{*}\right) \rightarrow 0,
$$

as $\kappa \rightarrow \infty$,

(ii)

$$
\int: \prod_{j=1}^{b} \phi^{\#}\left(x_{j}, 0\right)^{*} \prod_{j=1}^{b} \phi^{\#}\left(x_{j}, \varepsilon\right):\left[F_{l, 2 \varepsilon}^{\left(\kappa, \kappa^{\prime}\right)}\left(y ; \phi, \phi^{*}\right)-F_{l, 2 \varepsilon}\left(\underline{y} ; \phi, \phi^{*}\right)\right] d \mu_{C}\left(\phi, \phi^{*}\right) \rightarrow 0,
$$

as $\kappa, \kappa^{\prime} \rightarrow 0$, uniformly in $(\underline{x}, \underline{y})$.

Theorem II.1 is an immediate consequence of Proposition II.5.

Proof of Proposition II.5. We present the details of the proof of (II.35). The proof of (II.36) is identical and we omit it. Reasoning as in the proof of Proposition II.2 we reduce the proof to showing that

$$
\left\|F_{l, \beta}^{(\kappa)}\left(y ; \phi+\zeta f, \phi^{*}+\eta g\right)-F_{l, \beta}\left(y ; \phi+\zeta f, \phi^{*}+\eta g\right)\right\|_{L_{2}} \rightarrow 0,
$$

uniformly in $(\zeta, \eta) \in D_{r}$ and $(\underline{x}, \underline{y})$. Then

$$
\mathscr{F}^{(\kappa)}(\zeta, \eta ; \varrho) \rightarrow \mathscr{F}(\zeta, \eta, \varrho),
$$

uniformly in $(\zeta, \eta) \in D_{r}$. Here, $\mathscr{F}^{(\kappa)}(\zeta, \eta ; \varrho)$ is defined by (II.12) with $F_{l, \beta}$ replaced by $F_{l, \beta}^{(\kappa)}$. As a consequence, the derivatives of $\mathscr{F}^{(\kappa)}(\zeta, \eta ; \varrho)$ converge to the derivatives of $\mathscr{F}(\zeta, \eta ; \varrho)$, and the proof of (II.35) is complete.

To prove (II.37) we use the following uniform version of Lemma 3.5 of [10]:

Lemma II.7. Let $(\Omega, \mu)$ be a measurable space and $\left\{f_{n}^{\xi}\right\}_{n \geqq 1}$ and $f^{\xi}$ measurable functions depending on $\xi \in K, K$ compact, and such that

(i) $f_{n}^{\xi} \rightarrow f^{\xi}$ almost everywhere, uniformly in $\xi$,

(ii) $\left\|f_{n}^{\xi}\right\|_{L_{p}} \leqq C, p>1$, uniformly in $n$ and $\xi$.

Then $f^{\xi} \in L_{p}$ and $\left\|f^{\xi}-f_{n}^{\xi}\right\|_{L_{q}} \rightarrow 0$, uniformly in $\xi$, for all $1 \leqq q<p$.

By hypercontractivity [11], it is sufficient to show that

$$
\left\|F_{l, \beta}^{(\kappa)}\left(\underline{y} ; \phi+\zeta f, \phi^{*}+\eta g\right)\right\|_{L_{2}} \leqq C,
$$

uniformly in $\kappa, \underline{\underline{y}}$, and $(\zeta, \eta) \in D_{r}$, and

$$
F_{l, \beta}^{(\kappa)}\left(\underline{y} ; \phi+\zeta f, \phi^{*}+\eta g\right) \rightarrow F_{l, \beta}\left(\underline{y} ; \phi+\zeta f, \phi^{*}+\eta g\right),
$$

uniformly in $\underline{y}$ and $(\zeta, \eta) \in D_{r}$. 
To prove (II.39) we define $R^{(\kappa)}$ and $v^{(\kappa)}$ by formulas analogous to (II.18) and (II.21) with appropriate modifications. Then, repeating the arguments of the proof of Proposition II. 2 and noting that all the Feynman graph estimates are uniform in $\kappa$, we find

$$
\left\|F_{l, \beta}^{(\kappa)}\left(y ; \phi+\zeta f, \phi^{*}+\eta g\right)\right\|_{L_{2}} \leqq\left\|R^{(\kappa)}\right\|_{L_{2}} \leqq\|R\|_{L_{4}} \exp \left\|v-v^{(\kappa)}\right\|_{L_{4}} \leqq C,
$$

since $\left\|v-v^{(\kappa)}\right\|_{L_{4}} \leqq O\left(\kappa^{-\delta}\right)$ for $\kappa \geqq \kappa_{0}$ and some $\delta>0$.

Estimate (II.40) is a consequence of the following two estimates which can be easily established by methods of [5] (cf. Lemma II.3):

$$
\int\left\|\widetilde{K}_{l, \beta}\left(\phi+\zeta f, \phi^{*}+\eta g\right)-\tilde{K}_{l, \beta}^{(\kappa)}\left(\phi+\zeta f, \phi^{*}+\eta g\right)\right\|_{3}^{p} d \mu_{C}\left(\phi, \phi^{*}\right) \rightarrow 0
$$

uniformly for $(\zeta, \eta) \in D_{r}$, and

$$
\int\left|\tilde{\mathscr{A}}_{l, \beta}\left(\phi+\zeta f, \phi^{*}+\eta g\right)-\tilde{\mathscr{A}}_{l, \beta}^{(\kappa)}\left(\phi+\zeta f, \phi^{*}+\eta g\right)\right|^{p} d \mu_{C}\left(\phi, \phi^{*}\right) \rightarrow 0,
$$

as $\kappa \rightarrow \infty$, uniformly in $(\zeta, \eta) \in D_{r}$. These two estimates imply that the corresponding functions converge uniformly in $(\zeta, \eta)$ almost everywhere with respect to $d \mu_{c}$. Using Hölder continuity of $\operatorname{det}_{3}$, we infer (II.40).

\section{References}

1. Jaffe, A.: Heat kernel regularization and infinite dimensional analysis. In: Mathematical quantum field theory and related topics. Feldman, J., Rosen, L. (eds.). Canadian Mathematical Society Proceedings, Vol. 9. Providence, RI: AMS 1988

2. Glimm, J., Jaffe, A.: Quantum physics. Second Ed. Berlin, Heidelberg, New York: Springer 1987

3. Jaffe, A., Lesniewski, A.: Supersymmetric field theory and infinite dimensional analysis. Proceeding of the 1987 Cargèse Summer School. 't Hooft, G. et al. (eds.) (to appear)

4. Jaffe, A., Lesniewski, A., Weitsman, J.: The two-dimensional, $N=2$ Wess-Zumino model on a cylinder. Commun. Math. Phys. 112, 257-315 (1987)

5. Jaffe, A., Lesniewski, A.: A Priori estimates for the $N=2$, Wess-Zumino on a cylinder. Commun. Math. Phys. 114, 553-575 (1988)

6. Jaffe, A., Lesniewski, A., Weitsman, J.: The loop space $S^{1} \rightarrow \mathbb{R}$ and supersymmetric quantum fields. Ann. Phys. 183, 337-351 (1988)

7. Glimm, J., Jaffe, A.: Quantum field theory models. In: Statistical mechanics. De Witt, C., Storal, R. (eds.). New York: Gordon and Breach 1971. Reprinted. In: Collected papers, Vol. I: Quantum field theory and statistical mechanics; expositions. Boston: Birkhäuser 1985

8. Jaffe, A., Lesniewski, A., Weitsman, J.: Pfaffians on Hilbert space. J. Funct. Anal. (to appear)

9. Seiler, E.: Schwinger functions for the Yukawa model in two dimensions with space-time cutoff. Commun. Math. Phys. 42, 163-182 (1975)

10. Seiler, E., Simon, B.: Nelson's symmetry and all that in the Yukawa ${ }_{2}$ and $\phi_{3}^{4}$ field theories. Ann. Phys. 97, 470-518 (1976)

11. Nelson, E.: Probability theory and Euclidean field theory. In: Constructive quantum field theory. Velo, G., Wightman, A. (eds.). Berlin, Heidelberg, New York: Springer 1973

Communicated by A. Jaffe

Received August 8, 1988; in revised form October 16, 1988 